Int. J. Dev. Biol. 61: 303-310 (2017)

doi: $10.1387 / \mathrm{ijdb} .160252 \mathrm{af}$

\title{
ADHFe1: a novel enzyme involved in retinoic acid-dependent Hox activation
}

\author{
YEHUDA SHABTAI", NATALIE SHUKRUN" and ABRAHAM FAINSOD* \\ Department of Developmental Biology and Cancer Research, Institute for Medical Research Israel-Canada, \\ Faculty of Medicine, The Hebrew University of Jerusalem, Israel
}

\begin{abstract}
Retinoic acid (RA) signaling is a central pathway regulating anterior-posterior patterning of the embryo through its targets, the Hox genes. RA is produced by two sequential oxidations from vitamin A (retinol) and this biosynthesis has to be regulated temporally, spatially and quantitatively. Mining Xenopus embryonic expression databases identified a novel component of the RA metabolic network, ADHFe1. Using Xenopus laevis embryos as our experimental system we determined the temporal and spatial pattern of AdhFe1 expression. Gain- and loss-of-function of ADHFe1 were induced to study its function and the regulation of the AdhFe1 gene by RA was studied. Expression analysis localized the ADHFe1 protein to the late Spemann's organizer, the trunk organizer. Subsequently, ADHFe1 can be detected in the prechordal mesoderm, the notochord and the lateral plate mesoderm. Manipulation of ADHFe1 levels affects the normal Hox gene expression. The effects of ADHFe1 manipulation can by rescued by increasing the levels of RA or its biosynthesis. Expression of the AdhFe1 gene is regulated by RA itself. ADHFe1 is an enzyme active already during gastrula stages and the protein is still present during neurula stages. ADHFe1 regulates the expression of the Hox genes during the early patterning of the trunk. The effect of ADHFe1 on Hox expression is mediated through regulation of RA levels. ADHFe1 probably reduces retinaldehyde to retinol thereby restricting the availability of retinaldehyde, the substrate needed by retinaldehyde dehydrogenases to produce $\mathrm{RA}$ making it a novel regulator of RA concentrations in the embryo and RA homeostasis.
\end{abstract}

KEY WORDS: anterior-posterior patterning, Xenopus, alcohol dehydrogenase, hydroxyacid-oxoacid transhydrogenase

\section{Introduction}

Retinoic acid (RA) signaling is one of the central regulatory pathways active during early embryogenesis. It plays a pivotal role in patterning the embryo along the anterior-posterior axis by dose-dependent activation of Hox genes as well as by regulating somitogenesis. As such, this signaling pathway becomes activated in multiple sites during embryogenesis and in multiple instances in parallel. This parallel activity in different tissues and cell types, under differing conditions requires precise spatial, temporal and quantitative regulation. Several basic regulatory mechanisms cooperate to achieve this goal. RA signaling itself regulates the expression of multiple enzymes involved in RA biosynthesis and catabolism (Kedishvili, 2013). Enzymes like CYP26 of the cyto- chrome 4450 family metabolize RA to render it biologically inactive and promote its degradation (Kedishvili, 2013). Also, expression of the nuclear receptors mediating the gene regulatory effects of RA, i.e. retinoic acid receptors (RAR) and retinoid $X$ receptors (RXR), is tightly regulated in time and space (Dollé, 2009).

RAis produced from vitamin $\mathrm{A}$ (retinol, $\mathrm{ROL}$ ) through two sequential oxidation steps (Duester, 1996; Kedishvili, 2013). The first oxidation reaction is catalyzed by enzymes with alcohol dehydrogenase $(\mathrm{ADH})$ activity and produces retinaldehyde (RAL). Subsequently,

Abbreviations used in this paper: $\mathrm{ADH}$, alcohol dehydrogenase; $\mathrm{ADHFE}$, Fe-containing alcohol dehydrogenase 1; ALDH, aldehyde dehydrogenase; AP, anterior-posterior; MO, morpholino oligonucleotide; RA, retinoic acid; RAL, retinaldehyde; ROL, retinol; SDR, shot-chain dehydrogenase/reductase. *Address correspondence to: Abraham Fainsod. Department of Developmental Biology and Cancer Research, Institute for Medical Research Israel-Canada, Faculty
of Medicine,The Hebrew University of Jerusalem, Ein Kerem, Jerusalem 9112102, Israel. E-mail: abrahamf@ekmd.huji.ac.il - (D) http://orcid.org/0000-0002-6368-0416

\#Note: The indicated authors contributed equally to this paper.

Supplementary Material (one figure) for this paper is available at: http://dx.doi.org/10.1387/ijdb.160252af

Accepted: 10 August 2016.

ISSN: Online 1696-3547, Print 0214-6282

() 2017 UPV/EHU Press

Printed in Spain 
the RAL is oxidized by enzymes with aldehyde dehydrogenase (ALDH) activity to produce RA. The main members of the ALDH family producing RA are also known as retinaldehyde dehydrogenases (RALDH). The oxidation of ROL to RAL is performed by two families of enzymes, either members of the medium-chain ADH family or enzymes belonging to the short-chain dehydrogenase/ reductase (SDR) family (Duester, 1996; Kedishvili, 2013).

During embryogenesis the activation of RA signaling correlates with the zygotic expression of the Raldh2 gene (Ang, Duester, 1999; Chen et al., 2001). In Xenopus embryos Raldh2 mRNA initially localizes to Spemann's organizer on the dorsal lip of the blastopore (Chen et al., 2001). The early requirement for RALDH2 activity is supported by the observation that mouse mutants in the Raldh2 gene die soon after gastrulation (Niederreither et al., 1999). In line with this reasoning, it has been shown that during late blastula stages the embryo is primed to activate RA signaling but still requires a RALDH activity (Ang, Duester, 1999). Which enzymes are involved in the ROL to RAL oxidation reaction is less clear. Studies in a number of vertebrate model systems have identified the SDRs, RDH10 and RDHE2 as endogenous retinol dehydrogenases that provide an early activity required to produce RAL(Belyaeva et al., 2012; Rhinn et al., 2011). In Xenopusembryos, $R d h 10$ is expressed in a dorsal domain that partially overlaps with the domain of Raldh2expression (Strate et al., 2009). Rdhe2 is only zygotically transcribed from neurula stages onwards and does not qualify for the early function during gastrulation (Belyaeva et al., 2012). Another member of the SDR family, DHRS3 (retSDR), has been described that overlaps both spatially and temporally in its expression with RALDH2 and RDH10 (Kam et al., 2010). DHRS3 has been shown to play an important regulatory role by attenuating or restricting the RA signal (Billings et al., 2013; Feng et al., 2010). This enzyme mainly reduces RAL to ROL thus regulating the substrate availability for RALDH enzymes.

In order to identify additional enzymes possibly involved in gastrula stage RA metabolism, we searched the available Xenopus gene expression databases (Xenbase and others). We identified ADHFe1, as an enzyme expressed during mid gastrula stages beginning at stage 11 and continuing through neurula stages. Xenopus ADHFe1 protein and transcripts initially localize to the dorsal lip of the blastopore. Following invagination, they extend along the dorsal midline. During neurula stages the protein also localizes to the lateral plate mesoderm. Knock-down and overexpression experiments show that ADHFe1 is involved in the fine tuning of RA levels, using Hox gene expression as a readout. Loss-of-function resulted in upregulation of Hox transcription while ectopic expression compromised Hox activation. These results demonstrate that ADHFe1 is involved in RA-dependent anterior-posterior (AP) pattern formation, probably by performing the reduction of RAL to ROL, i.e.

A
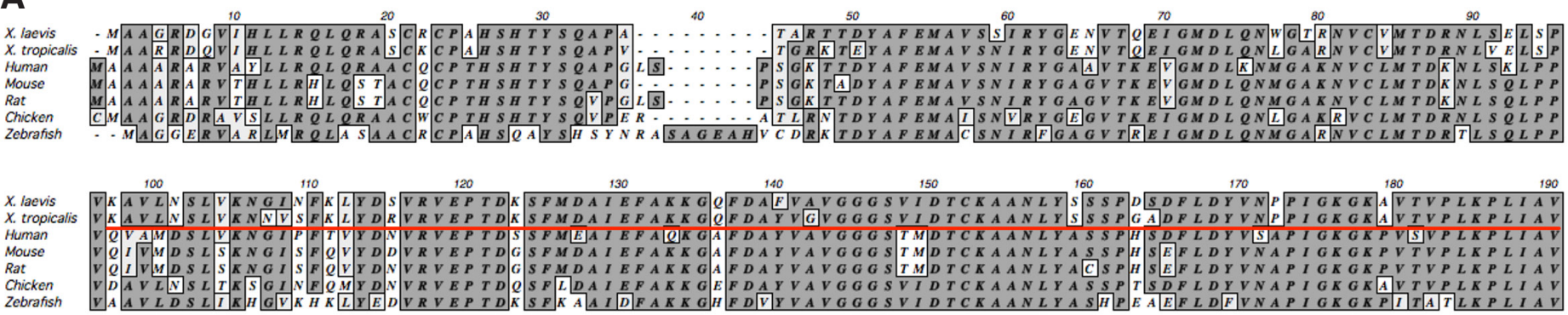

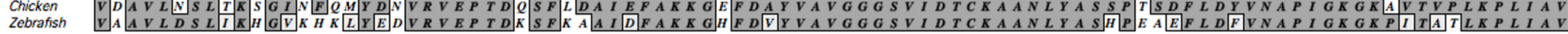
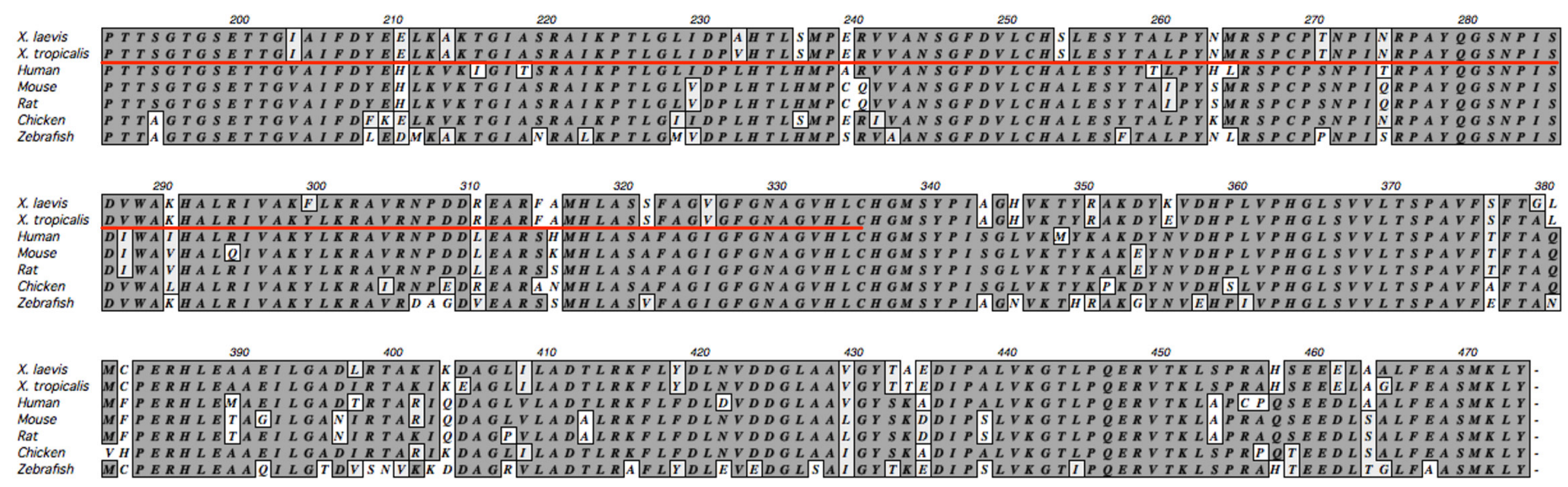

B

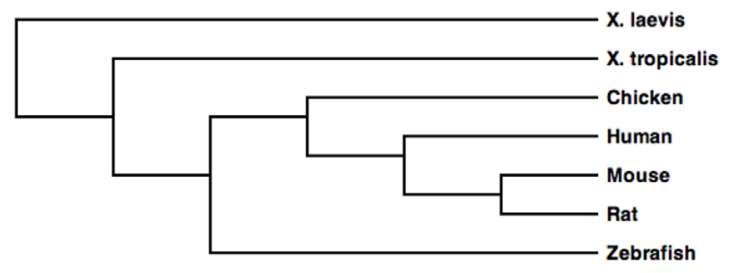

$\%$ Identity to $\mathbf{X}$. laevis

100

96.5

92.1

88.4

90.8

90.4

85.6
Fig. 1. Xenopus laevis ADHFe1 is extensively conserved among vertebrates. The full length open reading frame encoded by the AdhFe1 gene was PCR cloned from embryonic Xenopus laevis RNA samples. (A) Protein sequence alignment of the $\mathrm{X}$. laevis ADHFe1 protein to its orthologs from other vertebrates. Identical protein sequences are in gray boxes. The red line marks the human ADHFe1 sequence used to raise the polyclonal antibody. (B) Phylogenetic tree of the ADHFe1 protein family among representative vertebrates. The percent sequence identity to the $X$. laevis ADHFe1 sequence are shown. 
by restricting the substrate availability for RALDH. Together, this work identified a novel regulatory component in the metabolism of RA during early embryogenesis.

\section{Results}

\section{Identification and cloning of ADHFe1}

In an effort to better understand the biosynthesis of RA during gastrulation, we focused our efforts on identifying members of the ADH and SDR enzyme families expressed from late blastula to early neurula stages. We performed extensive data mining of expression databases (Bowes et al., 2010; Tan et al., 2013; Yanai et al., 2011) to identify those members of the ADH and SDR families known to be expressed during these stages. Among the genes identified we found $A d h F e 1$ which encodes an enzyme that has never been linked to RA metabolism. To characterize this enzyme in the context of early embryogenesis, we PCR cloned the full length open reading frame of $A d h F e 1$ from embryonic Xenopus laevis RNA samples (Fig. 1A). The predicted Xenopus laevisADHFe1 protein is 463 amino acids in length. The Xenopus protein displays extensive homology to other vertebrate ADHFe1 family members (85\% to $96 \%$ protein sequence identity; Fig. 1A,B), demonstrating a common evolutionary origin of this subfamily of the alcohol dehydrogenases (Fig. 1B).

\section{Onset of embryonic ADHFe1 expression coincides with the main wave of Hox activation}

To begin the characterization of the AdhFe1 gene we determined its temporal pattern of expression during early embryogenesis by quantitative real-time PCR (qPCR). RNA samples were collected from multiple embryonic stages from 16-cell stage (stage 5 ) to the initial neural tube stage (stage 19)(Nieuwkoop, Faber, 1967). Our results show that robust $A d h F e 1$ transcription begins towards mid gastrula (stage 11; Fig. 2A). Expression of AdhFe1 peaks towards the end of gastrulation (stage 12.5) / beginning of neurulation (stage 13) and remains high throughout the rest of the stages tested. Before stage 11, AdhFe1 transcripts are basically undetectable. This temporal pattern of expression is in agreement with the parameters we employed to search the expression databases to identify putative novel components of the RA metabolic network. The expression of Rdh10 and Dhrs3 was also studied in the same samples. This comparative expression analysis revealed that both members of the SDR family were up-regulated at the onset of gastrulation before the initial transcription of AdhFe1 (Fig. 2A). Also, while $R d h 10$ and Dhrs3 are down-regulated towards the end of gastrulation, $A d h F e 1$ remains strongly expressed during neurula stages (Fig. 2A).

To determine the spatial pattern of ADHFe1 activity we focused on the localization of the protein. To that end, a polyclonal antibody raised against a highly conserved human ADHFe1 peptide was used (Fig. 1A). To test the cross-reactivity and specificity of the anti-ADHFe1 antibodies, we transfected HEK293 cells with a pCS2-XADHFe1 plasmid to induce the expression of Xenopus AdhFe1. Co-transfection with a GFP expression plasmid was used to monitor transfection efficiency. RNA and protein extracts were prepared from experimental and control groups. Immunodetection of the expressed Xenopus ADHFe1 revealed that the protein was only detectable in cells transfected with the pCS2-XADHFe1 plasmid and not in the control transfected sample (Fig. 3A). The protein detected had an estimated molecular weight of about $50 \mathrm{KD}$ which corresponds to the predicted size from the cDNA sequence. To further demonstrate that the protein detected is the product of the pCS2-XADHFe1 expression plasmid we analyzed the RNA produced in the transfected cells. For this reason, we analyzed by qPCR the total RNA of the transfected cells with primers specific for the Xenopus ADHFe1 transcript. The results show that only the cells transfected with the expression plasmid gave a robust signal while the control sample had no PCR product (Fig. 3B). These results together show that the antibody against the human ADHFe1 can robustly detect the Xenopus protein product.

Embryos from defined stages were stained with the anti-ADHFe1 antibody to determine the spatial pattern of ADHFe1 expression. During the initial stages of ADHFe1 expression (stage 11), the
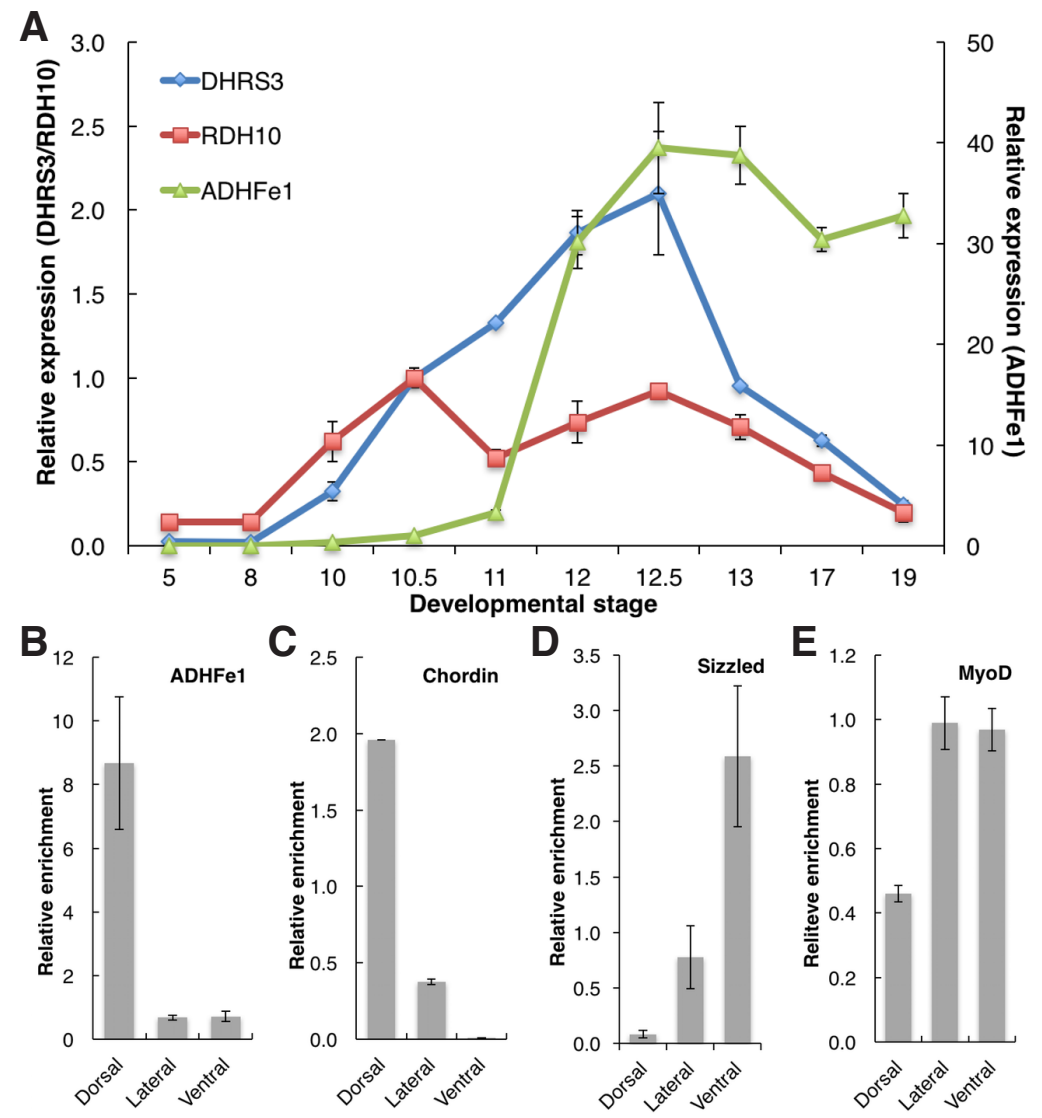

Fig. 2. ADHFe1 begins expression during mid gastrula in the late Spemann's organizer. (A) Expression of AdhFe1 was analyzed by qPCR using RNA samples from embryos of different developmental stages. The expression of AdhFe1 was compared to the temporal pattern of Rdh10 and Dhrs3 expression. The spatial distribution of AdhFe1 transcripts was determined by qPCR of RNA samples extracted from embryo fragments isolated from the dorsal, lateral and ventral regions of stage 12 embryos. (B). Marker gene expression was analyzed to determine the accuracy of the dissections. (C) Chordin expression was analyzed as a control for a dorsal-enriched gene. (D) Sizzled was utilized as a ventral-specific marker gene. (E) Expression of MyoD as a marker of the lateral and ventral regions. 
A

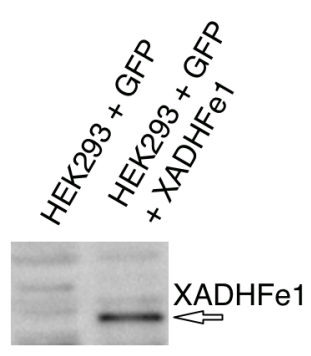

WB: anti- human ADHFe1

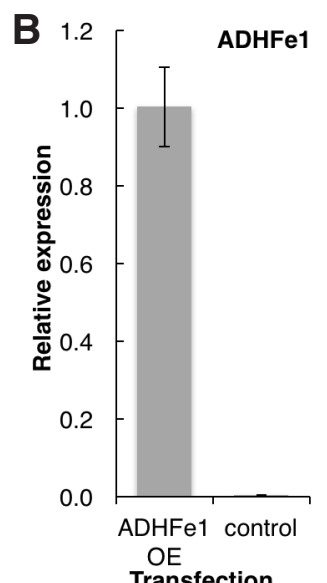

Transfection

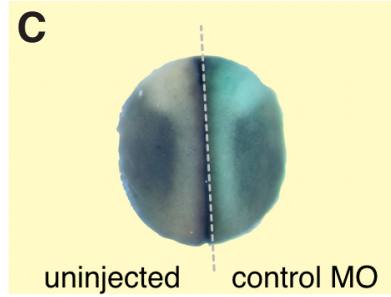

D

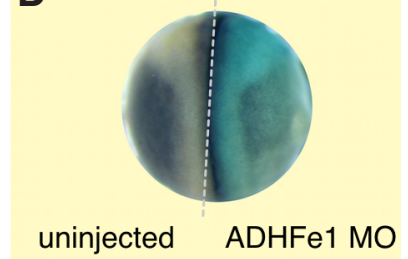

Fig. 3. A polyclonal antibody against human ADHFe1 detects the $\boldsymbol{X}$. laevis ADHFe1 protein. HEK293 cells were transfected with a XADHFe1 expression plasmid (pCS2$X A D H F e 1)$. All cells were co-transfected with a GFP expression plasmid to monitor the efficiency of transfection. (A) Protein extracts were prepared from the control (GFP only) and experimental samples and Western blot analysis was performed to detect the Xenopus ADHFe 1 protein. (B) RNA samples were prepared from the same transfected cells and analyzed by qPCR to demonstrate the expression of the $\mathrm{X}$. laevis AdhFe1 transcript. (C,D) Two-cell embryos were injected on one-side with the AdhFe1 antisense morpholino oligonucleotide (AdhFe1 MO; D) or with the control MO (C). The samples were co-injected with a lineage tracer. Embryos were processed for immunostaining for the endogenous ADHFe1 protein (purple) and the lineage tracer (turquoise). Dashed lines mark the boundary between the injected sides. protein encoded by this gene localized to the dorsal blastopore lip, i.e. the late Spemann's organizer (Fig. 4A). Towards the end of gastrulation (stage 12), ADHFe1 protein was found at the dorsal midline (Fig. 4B), suggesting that ADHFe1 positive cells invaginated during late gastrulation. To corroborate the dorsal midline expression of ADHFe1, we focused on localizing the mRNA of this gene. Stage 12 embryos were dissected into dorsal, lateral and ventral regions and RNA was prepared from each region. qPCR analysis with Xenopus AdhFe1 specific primers revealed that most transcripts of this gene localize to the dorsal region (Fig. 2B). Analysis of the localization of chordin, $M y o D$ and sizzled transcripts revealed the expected dorsal, lateral/ventral and ventral distribution of transcripts respectively (Fig. $2 \mathrm{C}-\mathrm{E}$ ). These results further support the dorsal localization of the ADHFe1 expression during gastrulation.

During mid neurula stages (stage 17), staining along the dorsal midline was still evident (Fig. 4D). Bisection of the embryos confirmed the localization of the ADHFe1 protein along the axial mesoderm, in the notochord and the prechordal mesoderm (PCM) (Fig. 4E). At stage 17 as well as stage 22, an additional ADHFe1 expression domain was detected (Fig. 4 C,D,F,G). This novel expression localized to the lateral plate mesoderm (LPM) as evidenced from the bisected embryos (Fig. 4H). Interestingly, this localization of the ADHFe1 protein abuts the CNS including the rostral region (Fig.
4C,D). Analysis of earlier developmental stages revealed that the LPM expression began with the transition from gastrula to neurula stages(stage 12; Fig. 4B).

\section{Developmental and biochemical functions of ADHFe1}

To investigate the biochemical function of ADHFe1 in the metabolism of RA we performed knock-down and gain-of-function experiments. To monitor the effect on RA biosynthesis, the changes in expression of 3' Hoxgenes, which are highly sensitive to RA levels, was determined (Cunningham, Duester, 2015). An antisense morpholino oligonucleotide (MO) was designed to knock-downADHFe1 levels (Heasman, 2002). To test the MO efficiency we constructed an expression plasmid with the AdhFe1 MO target sequence upstream of the GFP sequence. Embryos were co-injected with the $A D H F e 1$ target/GFP expression vector and the AdhFe1-specific $\mathrm{MO}$. At stage 11 the GFP-dependent fluorescence was analyzed (Suppl. Fig. S1). While all the embryos injected with the AdhFe1 MO lost fluorescence ( $n=20$; Suppl. Fig. S1F), all embryos co-injected with the control MO retained their fluorescence $(n=20$; Suppl. Fig. $\mathrm{S} 1 \mathrm{E})$. The fluorescence of embryos injected with a control GFP expression plasmid containing the Raldh2 MO target sequence was not affected by co-injection of the AdhFe1 MO ( $n=20$; Suppl. Fig. $\mathrm{S1C}$ ) or the control MO (Suppl. Fig S1B). These results strongly support the specificity of the AdhFe1 MO. To prove the MO specificity beyond doubt, ADHFe1 protein levels were analyzed in morphants by immunodetection. AdhFe1 MO was injected into one blastomere of the 2-cell embryo together with a lineage tracer. During neurula (stage 17), embryos were processed for immunodetection of the endogenous ADHFe1 protein (purple) and the lineage tracer (turquoise) (Fig. 3C,D). The ADHFe1 $M O$ drastically reduced the amount of ADHFe1 protein
Fig. 4. The ADHFe1 protein localizes to the dorsal midline and the lateral plate mesoderm. The ADHFe1 protein was localized in embryos from various stages utilizing the anti-ADHFe1 antibody. (A) Mid gastrula (stage 11), vegetal view. (B) Late gastrula (stage 12), vegetal view. (C) Late neural fold stage (stage 17), rostral view. (D) Stage 17, dorsal view. (E) Sagital bisected embryo, stage 17. (F) Stage 22, lateral view. (G) Stage 22, dorsal view. (H) Detail of cross section of stage 22 bisected embryo. st. 12

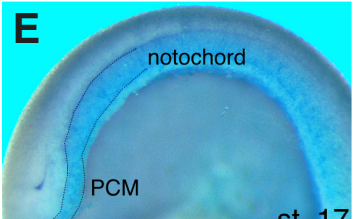

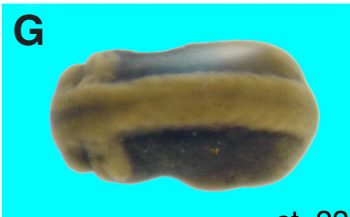

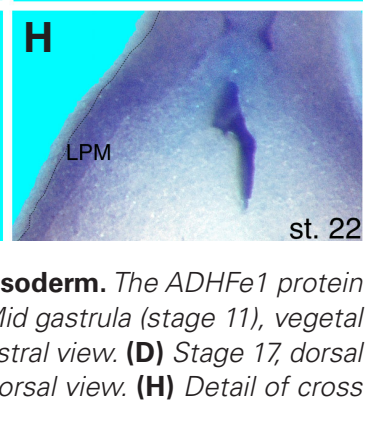



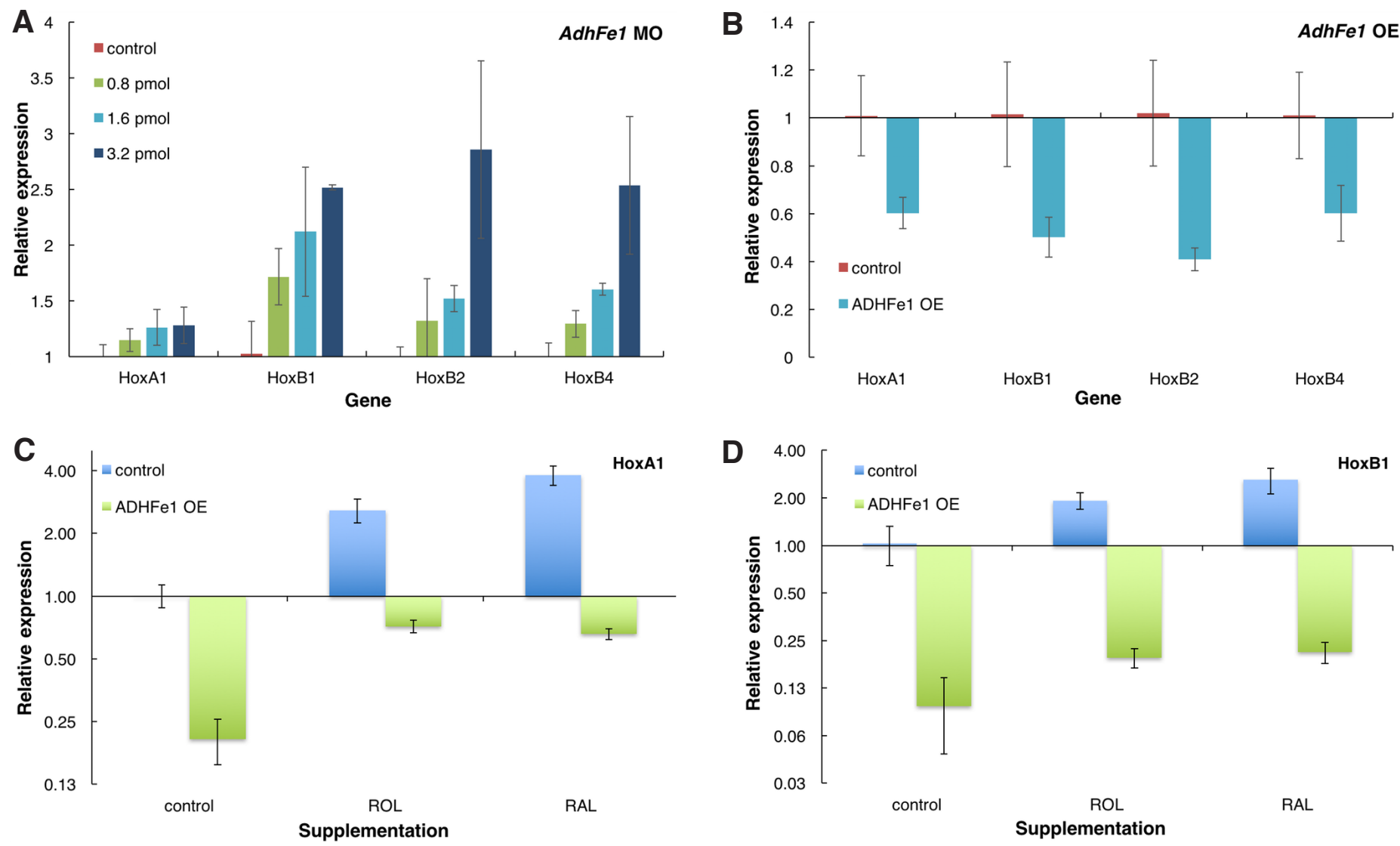

Fig. 5. ADHFe1 regulates Hox gene expression through retinoic acid signaling. In order to manipulate the level of ADHFe1 activity, embryos were injected radially with increasing concentrations of AdhFe1 MO (A) or the pCS2-XADHFe1 expression plasmid (B). The changes in Hox gene expression were determined by qPCR. To demonstrate the involvement of RA signaling in the function of ADHFe1 we performed a rescue experiment. Embryos overexpressing ADHFe1 were also treated with the RA precursors, RAL (1 $\mu M)$ or ROL $(10 \mu M)$ and analyzed for changes in HoxA1 (C) and HoxB1 (D) expression by qPCR.

in the injected side (Fig. 3D) while injection of the control MO had no effect on the protein levels (Fig. 3C). These results demonstrate the specificity of the AdhFe1 MO and the anti-ADHFe1 antibody.

To characterize the function of ADHFe1 in the context of RA biosynthesis, embryos were injected with increasing amounts of $\mathrm{MO}$ (0.8-3.2 pmol) and the effect was studied by monitoring the change in Hox expression. All four Hox genes monitored, HoxA1, HoxB1, HoxB2 and HoxB4 exhibited a dose-dependent response to the AdhFe1 MO (Fig. 5A). Interestingly, all four Hox genes were up-regulated as a result of the ADHFe1 knock-down, suggesting that this enzyme functions primarily to reduce the level of RA in the embryo.

We turned to overexpression (OE) of AdhFe1 to corroborate the effect of this enzyme on Hox expression. Embryos were injected with the AdhFe1 expression plasmid (pCS2-XADHFe1; 160 ng/embryo) and RNA was prepared for qPCR analysis of Hox expression at stage 11. In agreement with the knock-down results, ADHFe1 OE

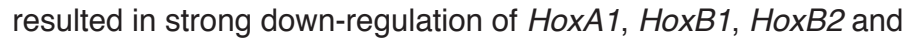
HoxB4 expression (Fig. 5B). This result further supports the notion that ADHFe1 activity promotes a decrease in RA levels.

To further explore this effect of ADHFe1 on the regulation of $\mathrm{Hox}$ gene expression and to determine whether it is mediated through RA signaling we designed rescue experiments. We reasoned that if $A D H F e 1$ regulated $R A$ levels by reducing $R A L$ to $R O L$, i.e. by reducing the substrate availability for RALDH enzymes, then supplementation with RA precursors should overcome this activity. To test this hypothesis, embryos were injected with the AdhFe1 expression plasmid and supplemented with either ROL $(10 \mu \mathrm{M})$ or RAL $(1 \mu \mathrm{M})$. Analysis of HoxA1 and HoxB1 expression showed that $\mathrm{ADHFe} 1 \mathrm{OE}$ alone resulted in strong down-regulation of their expression (Fig. 5C,D). Treatment of the control embryos with $\mathrm{ROL}$ or RAL, in contrast, resulted in the expected up-regulation in $\operatorname{HoxA1}$ and HoxB1 expression as a result of the promotion in RA biosynthesis (Fig. 5C,D). In agreement with our conclusion, supplementation with the RA precursors, RAL and ROL, overcame the down-regulatory effect of ADHFe1 OE, providing a partial rescue of expression levels (Fig. 5C,D). Together, these results strongly suggest that the effect of ADHFe1 on Hox expression is mediated through regulation of $\mathrm{RA}$ biosynthesis.

As mentioned above, RA feedback transcriptional regulation is a hallmark of genes encoding enzymes involved on RAmetabolism. To test whether this holds for $A d h F e 1$ as well, we determined if $A d h F e 1$ gene expression is also under RA regulation. The levels of RA in the embryo were manipulated in two ways: RA biosynthesis was compromised by inhibiting RALDH with DEAB or citral. Increased RA levels were achieved by precursor supplementation (ROL or $\mathrm{RAL}$ ) or actual RA treatment. During mid and late gastrulation, the levels of AdhFe1 expression were determined by qPCR. To 
A

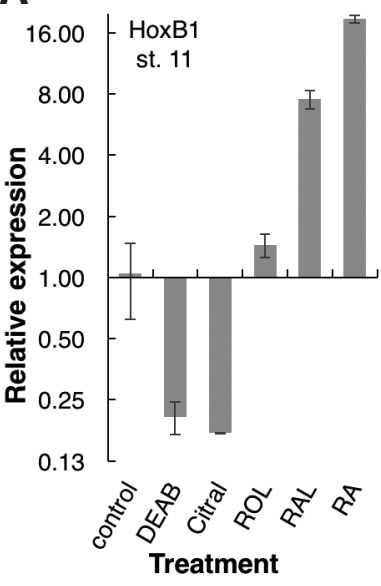

B

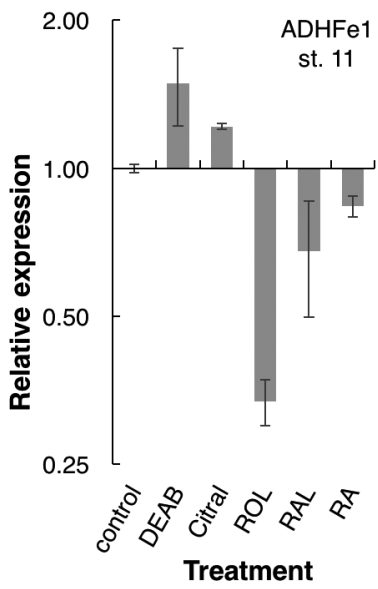

C

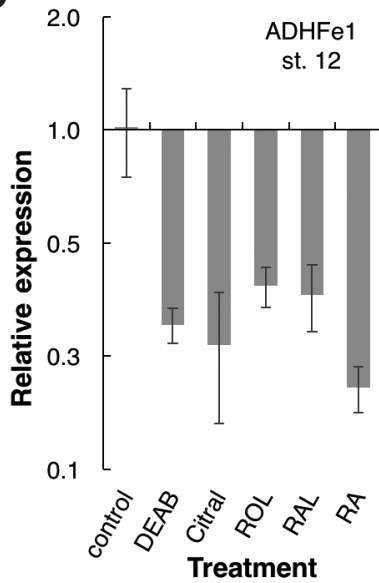

Fig. 6. AdhFe1 expression is negatively regulated by retinoic acid (RA). Embryos were treated with DEAB $(100 \mu \mathrm{M})$, citral (40 $\mu M)$ to inhibit the biosynthesis of $R A$ or with $R O L(10 \mu \mathrm{M}), \operatorname{RAL}(1 \mu \mathrm{M})$ and $R A(1 \mu \mathrm{M})$ to increase the levels of RA. All treatments were initiated during late blastula (stage 8) and analyzed by $q P C R$ for changes in expression. Analysis of $\mathrm{HoxB} 1$ expression at stage 11 was used as a control to monitor the efficiency of the treatments (A). AdhFe1 expression was determined during mid-(stage 11)(B) and late gastrula (stage 12)(C). asses the effect of the treatments on RA levels we determined the change in HoxB1 expression. Inhibition of RA biosynthesis with $\mathrm{DEAB}$ or citral severely reduced the expression of HoxB1 while ROL, RAL or RA supplementation increased the expression of this gene as expected (Fig. 6A). Analysis of AdhFe1 expression at stage 11 showed the inverse response to HoxB1 (Fig. 6B). This set of experiments suggest that RA signaling performs a negative regulatory role on AdhFe1 expression. Interestingly, when treated embryos were allowed to develop to late gastrula (stage 12) we identified a change in the regulation of $A d h F e 1$. Any treatment that changes the level of RA, negatively affected the expression of AdhFe1 (Fig. 6C) showing a simultaneous requirement for RA and negative regulation by high levels. Taken together, we have identified a novel enzyme involved in RA metabolism during early embryogenesis, which is required for the correct temporal and spatial induction of Hox gene expression, i.e. setup of the Hox code and the resulting anterior-posterior patterning of the embryo.

\section{Discussion}

In this study, we have demonstrated a novel function of ADHFe1 in the regulation of RA homeostasis during early embryonic development. ADHFe1 has thus far not been analyzed in the context of vertebrate embryogenesis. A number of studies are, however, available on the function of this enzyme in other contexts. ADHFe1 (EC 1.1.99.24) is a member of the alcohol dehydrogenase family of enzymes that are iron-activated. This enzyme is also known as an hydroxyacid-oxoacid transhydrogenase involved in the oxidation of 4-hydroxibutyrate in vertebrates (Kardon et al., 2006). Although only partially characterized, expression of the AdhFe1 gene has been linked to differentiation of adipose tissue (Kim et al., 2007). Multiple studies have identified the AdhFe1 promoter as undergoing hypermethylation in colorectal carcinomas (Naumov et al., 2013) and its expression level was positively correlated to the differentiation state of colorectal tumors (Tae et al., 2013). These additional studies propose a link between ADHFe1 activity levels and the differentiation state of cells and tissues. Our work identifies ADHFe1 activity very early during embryogenesis when the germ layers begin to differentiate and patterning along the early embryonic axes takes place.

The initial localization of the AdhFe1 transcripts and protein product to the late Spemann's organizer is of particular interest as this region acts as the so-called "trunk-tail organizer" (Niehrs, 2004). The trunk organizer is responsible for the formation and specification of embryonic regions caudal to the head domain. The vertebrate trunk domain is one of the main regions patterned by differential Hox gene activity (Durston, Zhu, 2015; Gaunt, 2015). Our results show that manipulation of ADHFe1 activity levels leads to abnormal expression of the Hox genes. Therefore, expression of AdhFe1 in the trunk organizer suggests a regulatory interaction with the Hox genes, a conclusion supported by our experiments. Future work will show to what extend the realization of the Hox code is dependent on ADHFe1, whether this enzyme merely fine-tunes RA levels or whether it may play a more active role in setting up the correct RA concentration along the AP axis.

Although AdhFe1 has been linked to a number of disease and differentiation processes, its biochemical function has only been partially characterized. The regulatory interaction between ADHFe1 activity and Hox expression levels provides an important insight for a novel activity of this enzyme. The Hox genes are some of the best characterized downstream targets of RA signaling (Parker et al., 2016). The changes in Hox gene expression following manipulation of ADHFe1 activity levels are consistent with changes in RA levels in the embryo. Although ADHFe1 has been described as an hydroxyacid-oxoacid transhydrogenase (Kardon et al., 2006), a role as an $\mathrm{NAD}(\mathrm{P})^{+}$-dependent alcohol dehydrogenase has also been proposed (Deng et al., 2002). As such, based on substrate specificity, this enzyme could perform the oxidation of ROL to RAL. Surprisingly, the response of the Hox genes to ADHFe1 manipulation was consistent with the opposite, a reduction in RA levels. This observation suggests that ADHFe1 also catalyzes the reduction of RAL to ROL, at least in the context of the early embryo. This conclusion was further supported experimentally by demonstrating that increasing ROL or RAL levels in the embryo rescued the effects of $A d h F e 1$ overexpression. These results link ADHFe1 to RA biosynthesis and suggests that this enzyme plays a role in the homeostatic regulating of RA levels.

Our results place ADHFe1 as a regulator of RA levels specifically during gastrula stages. The reduction of RAL to ROL would result in a reduced substrate availability for the RALDH, the enzyme which oxidizes RAL to RA (Shabtai et al., 2016). We have recently demonstrated that RA is not impacting on RALDH2 activity, i.e. that this enzyme, which performs the last step in RA biosynthesis, is devoid of a negative feedback regulatory loop (Shabtai et al., 
2016). RA-dependent regulation of ADHFe1 instead would place the RA-dependent feedback inhibition of the pathway at another step, one level up from RALDH2 itself.

Two members of the RALDH family are known to be expressed in Spemann's organizer during gastrula stages, Raldh2and Raldh3 (Chen et al., 2001; Lupo et al., 2005). Two additional members of the RAmetabolic network have been described in this region, Rdh10 and Dhrs3 (Kam et al., 2010). DHRS3 is of particular interest as it has been shown to perform a homeostatic regulatory function on RA levels (Feng et al., 2010) similar to the one we propose here for ADHFe1. Together, these data suggest that RA biosynthesis is under tight enzymatic regulation to achieve accurate signaling levels. Dissection of individual enzyme contributions necessary to achieve robust RA-dependent embryonic patterning awaits further characterization in vertebrate embryos.

\section{Materials and Methods}

\section{Embryo culture and treatments}

Xenopus laevis frogs were purchased from NASCO (Fort Atkinson, $\mathrm{WI})$. Experiments were performed after approval and under supervision of the Institutional Ethics Committee. Embryos were obtained by in vitro fertilization, incubated in $0.1 \% \mathrm{MBSH}$ and staged according to Nieuwkoop and Faber (1967). For microinjection, 2- to 4-cell embryos were injected radially or on one side. For lineage tracing the injected material was coinjected with FITC-dextran $\left(M_{r} 70,000\right) 10$ pg/injection (Sigma), subsequently detected either microscopically or with anti-fluorescein antibodies (1:5000, Anti-Fluorescein-AP Fab fragments, Roche).

Embryos were dissected into 3 parts: dorsal (DMZ), lateral (LMZ) and ventral marginal zone (VMZ) at stage 12, in 1\% MBSH buffer. Embryonic regions (15-20 embryos for each region) were then processed for RNA extraction and qPCR. ROL, RAL, RA, 4-diethylaminobenzaldehyde (DEAB) and 3,7-dimethyl-2,6-octadienal (citral) were purchased from Sigma-Aldrich, and stock solutions were prepared in DMSO.

\section{Cloning and sequencing of Xenopus AdhFe1}

The Xenopus AdhFe1 sequence was identified in the Xenbase database (Xenbase.org) by comparing the human AdhFe1 coding region (NM_144650.2) against the genome sequence of Xenopus laevis (V9.1 $\mathrm{J}$-strain). This sequence was used to design primers that were then used to amplify full-length AdhFe 1 from a stage 17 Xenopus laevis RNA (iScript cDNA kit; BioRad). The PCR product was cloned into the pCS2 vector and sequenced on both strands. Recently a provisional record for Xenopus AdhFe1 was released in GenBank (NM_001127802.1) Primers used for cloning: forward, 5'- ACAGAGGTGTTTTATCGCGATGGCTGCCG -3'; and reverse, 5' - GCATGTTCTTGAATGGATCGACAACCAATGTGC -3'. The phylogenetic tree analysis was conducted using the phylogenyfunction within the MacVector software package analysis tools using the neighborjoining method (Saitou, Nei, 1987).

\section{ADHFe1 knockdown}

Xenopus laevis ADHFe1 knockdown was induced by injection of a specific antisense morpholino oligonucleotide (XAdhFe1 MO), 5'- ACCGATTATACACGGATTGTGCTCA -3' (GeneTools). For controls, we also injected the Control Morpholino oligonucleotide (Control MO; GeneTools), 5'-CCTCTTACCTCAGTTACAATTTATA-3'.

\section{Quantitative real-time PCR}

Total RNA from embryos was extracted using a PerfectPure RNA Tissue Kit with DNase (5 Prime). Each experiment was repeated at least twice and involved at least five embryos/sample. cDNA was synthesized using the Iscript cDNA Synthesis kit (Bio-Rad). Quantitative real-time PCR (qPCR) was done in triplicate in a CFX384 Touch Real-Time PCR Detection System
TABLE 1

\section{qPCR PRIMER SEQUENCES}

\begin{tabular}{lll} 
Gene & Forward primer & Reverse primer \\
\hline AdhFE1 & TTGATGCCTTTGTTGCTGTT & CTGCTTTGCCTTCCCTATT \\
Dhrs3 & CAGGCGCAAGAAATCCTAAG & CAAAGGCCACGTTACAGGAT \\
Rdh10 & TTTGAAGCTGTGGTCTGCAT & GCCTGTTTCCTTTGAGCAAT \\
HoxA1 & CATCGCCTCGTCTGTGGT & GTCAGGTCCGTATGAATGGTG \\
HoxB1 & TCCCCCTCCAACAACAAACC & TTGCCCCAGTGCCAATGAC \\
HoxB2 & GCAGAATAGAGGTGGGTAGCA & GTGTAGGCAGGAGTCTGTTGAG \\
HoxB4 & CCAAGGATCTGTGCGTCAA & GCAGGATGGAGGCGAACT \\
Chordin & ACTGCCAGGACTGGATGGT & GGCAGGATTTAGAGTTGCTTC \\
Sizzled & AACAAGGTCTGCTCCTTCCA & CTGTGGGTCTGGTCCG \\
MyoD & CCCTGTTTCAATACCTCAGACAT & CGTGCTCATCCTCGTTATGG \\
GAPDH & GCTCCTCTCGCAAAGGTCAT & GGGCCATCCACTGTCTTCTG \\
\hline
\end{tabular}

(Bio-Rad). iTaq Universal SYBR Green Master Supermix (BioRad) was used. Results were calculated using the CP method. All qPCR primers used are listed in Table 1.

\section{Western blot analysis}

A rabbit polyclonal antibody raised against amino acids 110-335 of the human ADHFe1 (PA5-31416; Thermo Fisher Scientific) was used to detect the Xenopus laevis protein. Protein lysates for Western blots were prepared by homogenizing embryos or HEK293 cells in ice-cold lysis buffer (10 mM Tris-Cl pH 8.0, 1 mM EDTA, 0.5 mM EGTA, 1\% Triton X-100, $0.1 \%$ sodium deoxycholate, $0.1 \% \mathrm{SDS}, 140 \mathrm{mM} \mathrm{NaCl}$ ) supplemented with protease inhibitors ( $1 \mathrm{mM}$ phenylmethyl sulfonyl fluoride, $1 \mathrm{mM}$ pepstatin, and $10 \mu \mathrm{g} / \mathrm{ml}$ aprotinin). Homogenates were cleared by centrifugation at $14,000 \mathrm{rpm}$ for $10 \mathrm{~min}$ at $4^{\circ} \mathrm{C}$. Sodium dodecyl sulfate (SDS) sample buffer was added to the cleared lysate and boiled for 4 min before separation by SDS-polyacrylamide gel electrophoresis. Variability in cell densities was minimized by normalization to total protein concentration measured with the protein assay kit (Bio-Rad). Proteins were blotted to polyvinylidene difluoride membranes (Bio-Rad), and blots were blocked in 5\% milk in TBS $+0.1 \%$ Tween and probed with anti-ADHFe1 antibody (1:10000) for $1 \mathrm{hr}$ at room temperature and horseradish peroxidase-conjugated goat anti-rabbit lgG (1:20000; A0545, Sigma) as a second antibody. Immunoreactive proteins were detected using a chemiluminescence kit (Biological Industries) according to the manufacturer's protocol.

\section{Whole mount antibody staining}

Whole mount antibody staining was performed as described previously (Sive et al., 2000). Embryos were incubated with anti-human ADHFe1 rabbit polyclonal antibody (1:500; PA5-31416; Thermo Fisher Scientific) and alkaline phosphatase-conjugated goat anti-rabbit IgG (1:5000; A3687, Sigma) as a second antibody. The localization was visualized in BM-Purple solution (Roche). When needed, we performed a second staining against the FITC molecules using an anti-FITC antibody (1:10000; Roche) and stained using BCIP.

\section{Cell culture and transfection}

HEK293 cells were grown in DMEM supplemented with $10 \%$ fetal calf serum, at $37^{\circ} \mathrm{C}$ and $5 \% \mathrm{CO} 2$ atmosphere. For transfection, cells were plated on a 6-well plate, grown to $50 \%$ confluence and transfected using polyethyleneimine (PEI; Sigma). Briefly, $2 \mu \mathrm{g}$ of DNA were used per well mixed with $\mathrm{PEI}$ to a ratio of 1:5 respectively. Cells were co-transfected with $1 \mu \mathrm{g}$ of each plasmid (pCS2-AdhFe1 and pCS2-GFP). Transfection efficiency of transiently transfected cells was routinely $70 \%$ or more estimated by microscopy. After 48 hours, cells were harvested and lysed for qPCR or Western blot analysis.

\section{Acknowledgements}

This work was supported in part by grants from the Canadian Friends 
of the Hebrew University; the Manitoba Liquor Control Commission [grant number RG-003-14 (to A.F.)]; Canadian Institutes of Health Research [grant number TEC-128094 (to A.F.)]; and the Chief Scientist of the Israel Ministry of Health [grant number 3-10068 (to A.F.)].

\section{References}

ANG HL, DUESTER G (1999). Stimulation of premature retinoic acid synthesis in Xenopus embryos following premature expression of aldehyde dehydrogenase ALDH1. Eur J Biochem 260: 227-234.

BELYAEVA OV, LEE S-A, ADAMS MK, CHANG C, KEDISHVILI NY (2012). Short chain dehydrogenase/reductase rdhe2 is a novel retinol dehydrogenase essential for frog embryonic development. J. Biol. Chem. 287: 9061-9071.

BILLINGS SE, PIERZCHALSKI K, BUTLER TJADEN NE, PANG X-Y, TRAINOR PA, KANE MA, MOISE AR (2013). The retinaldehyde reductase DHRS3 is essential for preventing the formation of excess retinoic acid during embryonic development. FASEB J. 27: 4877-4889.

BOWES JB, SNYDER KA, SEGERDELL E, JARABEK CJ, AZAM K, ZORN AM, VIZE PD (2010). Xenbase: gene expression and improved integration. Nucleic Acids Res 38: D607-12.

CHEN Y, POLLET N, NIEHRS C, PIELER T (2001). Increased XRALDH2 activity has a posteriorizing effect on the central nervous system of Xenopus embryos. Mech Dev 101: 91-103.

CUNNINGHAM TJ, DUESTER G (2015). Mechanisms of retinoic acid signalling and its roles in organ and limb development. Nat Rev Mol Cell Biol 16: 110-123.

DENG Y, WANG Z, GU S, JI C, YING K, XIE Y, MAO Y (2002). Cloning and characterization of a novel human alcohol dehydrogenase gene (ADHFe1). DNA Seq 13: 301-306

DOLLÉ P (2009). Developmental expression of retinoic acid receptors (RARs). Nucl Rec Sig 7: e006.

DUESTER G (1996). Involvement of alcohol dehydrogenase, short-chain dehydrogenase/reductase, aldehyde dehydrogenase, and cytochrome P450 in the control of retinoid signaling by activation of retinoic acid synthesis. Biochemistry 35: $12221-12227$.

DURSTON AJ, ZHU K (2015). A time space translation hypothesis for vertebrate axial patterning. Semin Cell Dev Biol 42: 86-93.

FENG L, HERNANDEZ RE, WAXMAN JS, YELON D, MOENS CB (2010). Dhrs3a regulates retinoic acid biosynthesis through a feedback inhibition mechanism. Dev Biol 338: 1-14.

GAUNTSJ (2015). The significance of Hox gene collinearity. Int J Dev Bio/59:159-170.

HEASMAN J (2002). Morpholino oligos: making sense of antisense? Dev Biol 243: 209-214.

KAM RKT, CHEN Y, CHAN S-O, CHAN W-Y, DAWID IB, ZHAO H (2010). Developmental expression of Xenopus short-chain dehydrogenase/reductase 3. Int $\mathrm{J}$ Dev Biol 54: 1355-1360.

KARDONT, NOËL G, VERTOMMEN D, VAN SCHAFTINGEN E (2006). Identification of the gene encoding hydroxyacid-oxoacid transhydrogenase, an enzyme that metabolizes 4-hydroxybutyrate. FEBS Letters 580: 2347-2350.
KEDISHVILI NY (2013). Enzymology of retinoic acid biosynthesis and degradation $J$ Lipid Res 54: 1744-1760.

KIM J-Y, TILLISON KS, ZHOU S, LEE JH, SMAS CM (2007). Differentiation-dependent expression of Adhfe1 in adipogenesis. Arch Biochem Biophys 464: 100-111.

LUPO G, LIU Y, QIU R, CHANDRARATNA RAS, BARSACCHI G, HE R-Q, HARRIS WA (2005). Dorsoventral patterning of the Xenopus eye: a collaboration of Retinoid, Hedgehog and FGF receptor signaling. Development 132: 1737-1748.

NAUMOV VA, GENEROZOV EV, ZAHARJEVSKAYANB, MATUSHKINA DS, LARIN AK, CHERNYSHOV SV, ALEKSEEV MV, SHELYGIN YA, GOVORUN VM (2013). Genome-scale analysis of DNA methylation in colorectal cancer using Infinium HumanMethylation450 BeadChips. Epigenetics 8: 921-934.

NIEDERREITHER K, SUBBARAYAN V, DOLLÉ P, CHAMBON P (1999). Embryonic retinoic acid synthesis is essential for early mouse post-implantation development. Nat Genet 21: 444-448.

NIEHRS C (2004). Regionally specific induction by the Spemann-Mangold organizer Nat Rev Genet 5: 425-434.

NIEUWKOOP PD, FABER J (1967). Normal table of Xenopus laevis (Daudin): A systematical and chronological survey of the development from the fertilized egg till the end of metamorphosis., 2nd ed. North-Holland Publishing Company, Amsterdam.

PARKER HJ, BRONNER ME, KRUMLAUF R (2016). The vertebrate Hox gene regulatory network for hindbrain segmentation: Evolution and diversification: Coupling of a Hox gene regulatory network to hindbrain segmentation is an ancient trait originating at the base of vertebrates. Bioessays 38: 526-538.

RHINN M, SCHUHBAUR B, NIEDERREITHER K, DOLLÉ P (2011). Involvement of retinol dehydrogenase $10 \mathrm{in}$ embryonic patterning and rescue of its loss of function by maternal retinaldehyde treatment. Proc Nat Acad Sci USA 108: 16687-16692.

SAITOU N, NEI M (1987). The neighbor-joining method: a new method for reconstructing phylogenetic trees. Mol Biol Evol 4: 406-425.

SHABTAI Y, JUBRAN H, NASSAR T, HIRSCHBERG J, FAINSOD A (2016). Kinetic characterization and regulation of the human retinaldehyde dehydrogenase 2 enzyme during production of retinoic acid. Biochem. J. 473: 1423-1431.

SIVE HL, GRAINGER RM, HARLAND RM (2000). Early development of Xenopus laevis: a laboratory manual. Cold Spring Harbor Laboratory Press, Cold Spring Harbor, NY.

STRATE I, MIN TH, ILIEV D, PERA EM (2009). Retinol dehydrogenase 10 is a feedback regulator of retinoic acid signalling during axis formation and patterning of the central nervous system. Development 136: 461-472.

TAE CH, RYU KJ, KIM S-H, KIM HC, CHUN H-K, MIN B-H, CHANG DK, RHEE P-L, KIM JJ, RHEE JC, KIM Y-H (2013). Alcohol dehydrogenase, iron containing, 1 promoter hypermethylation associated with colorectal cancer differentiation. $B M C$ Cancer 2009 9:383 13: 142

TAN MH, AU KF, YABLONOVITCH AL, WILLS AE, CHUANG J, BAKER JC, WONG WH, LIJB (2013). RNA sequencing reveals a diverse and dynamic repertoire of the Xenopus tropicalis transcriptome over development. Genome Res 23: 201-216.

YANAI I, PESHKIN L, JORGENSEN P, KIRSCHNER MW (2011). Mapping gene expression in two Xenopus species: evolutionary constraints and developmental flexibility. Dev Cell 20: 483-496. 


\section{Further Related Reading, published previously in the Int. J. Dev. Biol.}

The involvement of three signal transduction pathways in botryllid ascidian astogeny, as revealed by expression patterns of representative genes

Amalia Rosner, Gilad Alfassi, Elizabeth Moiseeva, Guy Paz, Claudette Rabinowitz, Ziva Lapidot, Jacob Douek, Abraham Haim and Baruch Rinkevich

Int. J. Dev. Biol. (2014) 58: 677-692

Retinoid signalling is required for information transfer from mesoderm to neuroectoderm during gastrulation

Ferran Lloret-Vilaspasa, Hans J. Jansen, Koen de Roos, Rosh A.S. Chandraratna, Maija H. Zile, Claudio D. Stern and Antony J. Durston Int. J. Dev. Biol. (2010) 54: 599-608

Developmental expression of Xenopus short-chain dehydrogenase/reductase 3

Richard K.T. Kam, Yonglong Chen, Sun-On Chan, Wood-Yee Chan, Igor B. Dawid and Hui Zhao Int. J. Dev. Biol. (2010) 54: 1355-1360

The expression of Stra6 and Rdh10 in the avian embryo and their contribution to the generation of retinoid signatures Susan Reijntjes, Maija H. Zile and Malcolm Maden

Int. J. Dev. Biol. (2010) 54: 1267-1275

Induction of intermediate mesoderm by retinoic acid receptor signaling from differentiating mouse embryonic stem cells

Shiho Oeda, Yohei Hayashi, Techuan Chan, Minoru Takasato, Yuko Aihara,Koji Okabayashi, Kiyoshi Ohnuma and Makoto Asashima

Int. J. Dev. Biol. (2013) 57: 383-389

Retinoic acid metabolizing factor XCyp26c is specifically expressed in neuroectoderm and regulates anterior neural patterning in Xenopus laevis

Misaki Tanibe, Tatsuo Michiue, Akira Yukita, Hiroki Danno, Masayuki Ikuzawa, Shoichi Ishiura and Makoto Asashima

Int. J. Dev. Biol. (2008) 52: 893-901

The importance of the posterior midline region for axis initiation at early stages of the avian embryo

Oded Khaner

Int. J. Dev. Biol. (2007) 51: 131-137

Early stages of neural crest ontogeny: formation and regulation of cell delamination Chaya Kalcheim and Tal Burstyn-Cohen

Int. J. Dev. Biol. (2005) 49: 105-116

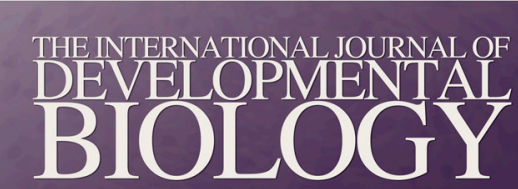

Volume 60 Nos. 10/11/12
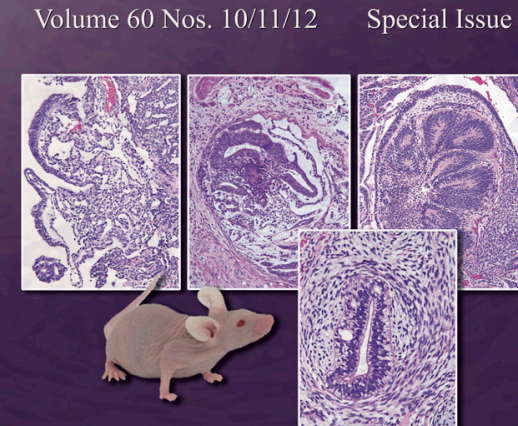

Atlas of Human Experimental Teratomas

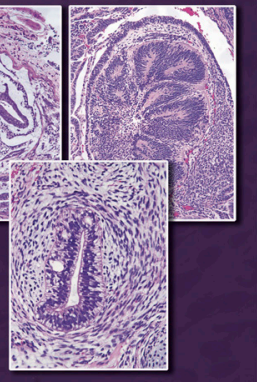

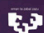

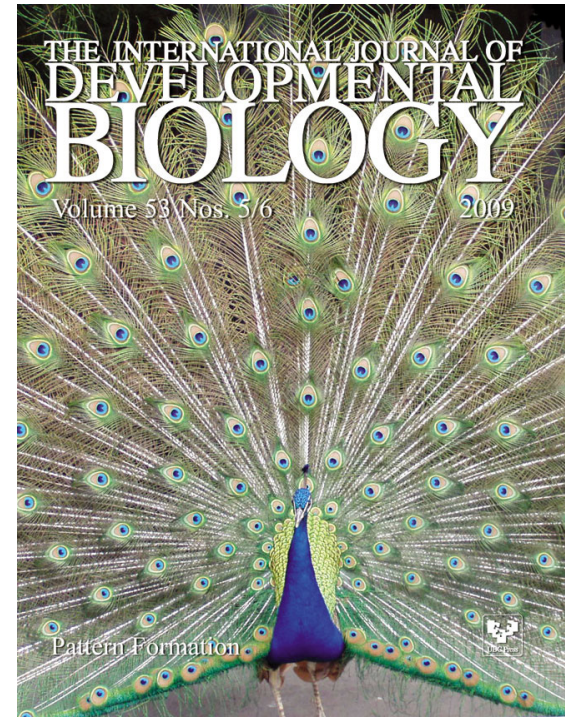

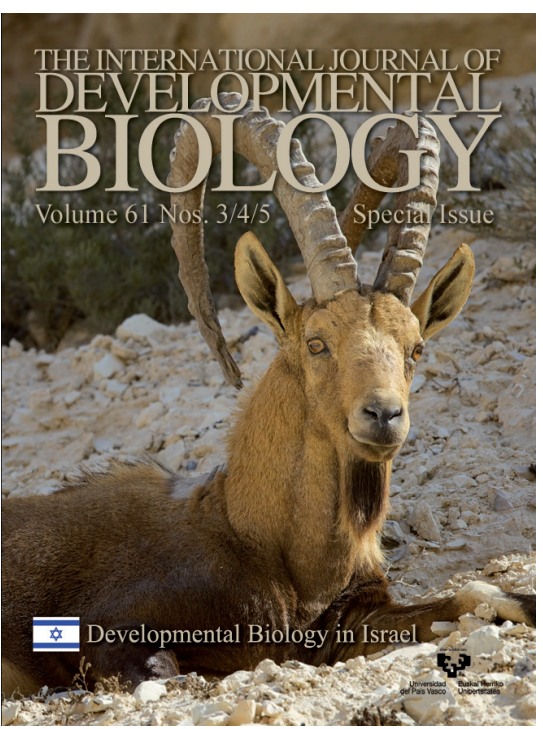

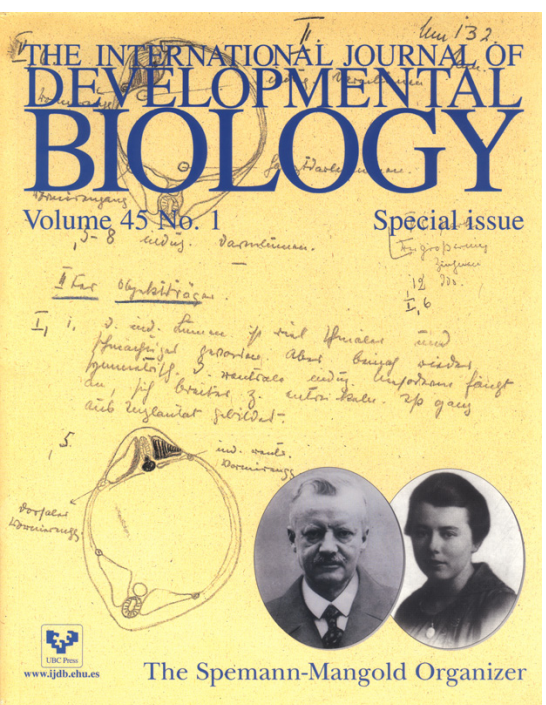

